\title{
Expression of Aquaporins in Human Embryos and Potential Role of AQP3 and AQP7 in Preimplantation Mouse Embryo Development
}

\author{
Yun Xionga,b,d Ya-Jing Tan ${ }^{a, b, d}$ Yi-Meng Xiong ${ }^{a, b}$ Yi-Ting Huang ${ }^{a, c}$ Xiao-Ling Hu $u^{a, c}$ \\ Yong-Chao Lu ${ }^{a, c}$ Ying-Hui Ye ${ }^{a, c}$ Ting-Ting Wang ${ }^{a, c}$ Dan Zhang ${ }^{a, c}$ Fan Jin ${ }^{a, c}$ \\ He-Feng Huanga,c Jian-Zhong Sheng ${ }^{a, b}$ \\ aKey Laboratory of Reproductive Genetics (Zhejiang University), Ministry of Education, Hangzhou, \\ China; 'bepartment of Pathology \& Pathophysiology, School of Medicine, Zhejiang University, \\ Hangzhou, China; 'Department of Reproductive Endocrinology, Women's Hospital, School of Medicine, \\ Zhejiang University, Hangzhou, China; ${ }^{\mathrm{Y}}$. X. and Y-J. T. contributed equally to this work
}

\section{Key Words}

Aquaporin $3 \cdot$ Aquaporin 7 • Early embryo $•$ Development

\begin{abstract}
Background/Aims: Water channels, also named aquaporins (AQPs), play crucial roles in cellular water homeostasis. Methods: RT-PCR indicated the mRNA expression of AQPs 1-5, 7, 9, and 11-12, but not AQPs $0,6,8$, and 10 in the 2 8-cell stage human embryos. AQP3 and AQP7 were further analyzed for their mRNA expression and protein expression in the oocyte, zygote, 2-cell embryo, 4-cell embryo, 8-cell embryo, morula, and blastocyst from both human and mouse using RT-PCR and immunofluorescence, respectively. Results: AQP3 and AQP7 were detected in all these stages. Knockdown of either AQP3 or AQP7 by targeted siRNA injection into 2-cell mouse embryos significantly inhibited preimplantation embryo development. However, knockdown of AQP3 in JAr spheroid did not affect its attachment to Ishikawa cells. Conclusion: These data demonstrate that multiple aquaporins are expressed in the early stage human embryos and that AQP3 and AQP7 may play a role in preimplantation mouse embryo development.
\end{abstract}




\section{Cellular Physiology and Biochemistry}

Cell Physiol Biochem 2013;31:649-658

DOI: $10.1159 / 000350084$

Published onlıne: May 06, 2013

(C) 2013 S. Karger AG, Basel

www.karger.com/cpb

650

\section{Introduction}

Water channels, also named aquaporins (AQPs), are a family of small (25-34 kDa), hydrophobic, integral membrane channel proteins that facilitate rapid, passive movement of massive amounts of water [1,2]. Thirteen isoforms of AQPs (AQP0-12) occur in mammals, and they comprise three major subfamilies: the aquaporin subfamily including AQP0, AQP1, $\mathrm{AQP2}, \mathrm{AQP} 4$ and AQP5, the aquaglyceroporin and glycerol facilitator (GlpF) subfamily including AQP3, AQP7, AQP9 and AQP10, and S-aquaporin subfamily including AQP6, AQP8, AQP11 and AQP12 [2-5]. AQPs 0-11 have been identified in both female and male reproduction systems [6].

A previous study showed that AQPs 1, 3 and 5-9 were expressed in mouse embryos [7]. However, another study did not detect AQPs 1, 2, 4, 5 and 6 in mouse oocytes and embryos at any stage [8]. As an aquaglyceroporin that is permeable to water and small neutral solutes including glycerol and urea [1-5], the functions of AQP3 in the transport of water and cryoprotectants in mouse oocytes and embryos have been investigated in several studies [9-11]. AQP3 facilitates water and glycerol diffusion in mouse morula [10, 12]. Increased expression of AQP3 in mouse oocytes by cRNA injection improves the survival of mouse oocytes after cryopreservation [11]. Decreased AQP3 expression was observed in oocytes of the mice treated with control ovarian stimulation, and this was associated with low fertilization rate [13]. AQP7 is another aquaglyceroporin expressed in mouse oocytes and embryos [7, 8]. Our recent study demonstrated that cryoprotectants induced AQP7 expression in mouse oocyte, and, this was associated with a low survival rate of vitrified oocytes during cryopreservation [14].

To investigate the expression profile of AQPs in human embryos, we examined mRNA expression of AQPs 0-12. We also examined the development of murine embryos following knockdown of AQP3 and AQP7, respectively.

\section{Materials and Methods}

\section{Embryo collection}

Human preimplantation embryos were donated by patients who had a successful pregnancy from an in vitro fertilization program at Women's Hospital, School of Medicine, Zhejiang University. Donation was voluntary and informed consent was given. This research was approved by the Ethics Committee for Research on Human Subjects of Zhejiang University. In order to exclude the effect of sperms that adhere to the zona pellucida (ZP), the embryos, that were used for RNA extraction, were dealt with Tyrode's solution, acidic (Sigma-Aldrich, St. Louis, MO, USA) to digest ZP.

Mouse oocytes and embryos were collected from female ICR mice (Shanghai Institutes for Biological Science, Shanghai, China), which were induced to superovulate with intraperitoneal injections of 10 IU of pregnant mare serum gonadotropin (PMSG) and $10 \mathrm{IU}$ of human chorionic gonadotropin (hCG) given $48 \mathrm{~h}$ apart, and were mated with male ICR mice. Twelve hours after hCG injection, the female mice were checked to see if there was vaginal plug. Unfertilized oocytes were collected from the ampullar portion of the oviducts at $14 \mathrm{~h}$ after hCG injection without mating. They were freed from cumulus cells by suspending them in HEPES-buffered human tubal fluid (HTF) (10 mM HEPES, Irvine Scientific, Santa Ana, CA, USA) containing $300 \mu \mathrm{g} / \mathrm{ml}$ hyaluronidase followed by washing with HEPES-buffered HTF. Zygote and two-cell embryos were flushed from the oviducts of mated mice with HEPES-buffered HTF 24-30 h after checking for a vaginal plug. Two-cell embryos were cultured in HTF medium containing $10 \%$ serum substitute supplement (Irvine Scientific) in a humidified incubator equilibrated with $5 \% \mathrm{CO} 2$ in air at $37^{\circ} \mathrm{C}$. Fourcell embryos, eight-cell embryos, morula and blastocysts were obtained by culturing two-cell embryos. All experiments were approved by the Animal Ethics Committee of Zhejiang University.

RT-PCR and quantitative real-time PCR ( $q P C R)$ analysis

Total RNA was extracted from 30 50 embryos from 2-cell to blastocyst stage using RNeasy Plus Micro Kit according to the manufacture's instructions (QIAGEN, Valencia, CA, USA). The cDNA was prepared by 


\begin{tabular}{|c|c|c|c|}
\hline $\mathrm{cDNA}$ & Primer set & Sense primer $\left(5^{\prime} \rightarrow 3^{\prime}\right)$ & Antisense primer $\left(5^{\prime} \rightarrow 3^{\prime}\right)$ \\
\hline \multirow[t]{2}{*}{ hAQP0 } & Outer set & CCTGCTCCGTGCCTTCTGCTAT & GCCATTCCGCCTCTCGTCGTAT \\
\hline & Inner set & CTGCTCCGTGCCTTCTGCTAT & CCTGTGACCTCTGGTTGTCCAT \\
\hline \multirow[t]{2}{*}{ hAPQ1 } & Outer set & GGCCAGCGAGTTCAAGAAGA & GGGCCAGGATGAAGTCGTAG \\
\hline & Inner set & GTCACACTGGGGCTGCTGCT & CCAGCACGCATAGCACCAGC \\
\hline \multirow[t]{2}{*}{ hAQP2 } & Outer set & GCGTTTGGCTTGGGTATTGG & GAGTGCAGCTCCACCGACTG \\
\hline & Inner set & AGCCGCTCTGCTCCATGAGATCAC & GGCGGAAACAGCACGTAGTTGTAG \\
\hline \multirow[t]{2}{*}{ hAQP3 } & Outer set & TCCTGGTGATGTTTGGCTGT & TACACGAAGACACCCGCAAT \\
\hline & Inner set & CAGACGCTGGGAGCCTTCTT & ACGGGGTTGTTGTAGGGGTC \\
\hline \multirow[t]{2}{*}{ hAQP4 } & Outer set & ATCAACTGGGGTGGAACAGA & AGCTTCCTTTTGTTTGCTGG \\
\hline & Inner set & CATCGCCAAGTCTGTCTTCTACA & GCTATTGAGCCAGTGACATCAGTC \\
\hline \multirow[t]{2}{*}{ hAQP5 } & Outer set & CACATCAACCCCGCCATCAC & TCCTCCCAGTCCTCGTCAGG \\
\hline & Inner set & АСTCTGCATCTTCGCCTCCA & AGGCTCAGGGAGTTGGGGAA \\
\hline \multirow[t]{2}{*}{ hAQP6 } & Outer set & CACCTTCAACCTGGTCACCG & CCCACCTCTACGGTGCCTGT \\
\hline & Inner set & GGGCTGCTCTGCTTTATGGG & TTCCCAATGATGATGGCAGG \\
\hline \multirow[t]{2}{*}{ hAQP7 } & Outer set & CGGAGGCTGAGAATCAGAG & СTCTGCTTAGAAGTGCTCTAGGG \\
\hline & Inner set & ATGATGGTATTCGGCCTTGGT & AGTGGAGAATGGCCGTGTAGA \\
\hline \multirow[t]{2}{*}{ hAQP8 } & Outer set & AGAATGGGACGGACACTGGG & ATGCAGCCTCCAGACACAGG \\
\hline & Inner set & GTGGACACTTCAACCCTGCG & ATGATCTCTGCCACCAACGC \\
\hline \multirow[t]{2}{*}{ hAQP9 } & Outer set & GCGGTGTCTCTGGTGGTCAC & TGACAGCACCAACCAAAGGG \\
\hline & Inner set & СTTATGTCCTTTGCTGGTGG & AGTCTGGGACTCAGGTCTCG \\
\hline \multirow[t]{2}{*}{ hAQP10 } & Outer set & AGTGACTGGCCCCAAGGAGA & ACCACCAGCCATTACCAGCA \\
\hline & Inner set & TAGCCATCTACGTGGGTGGT & TGGTAAGTGGCTGTGCCAAC \\
\hline \multirow[t]{2}{*}{ hAQP11 } & Outer set & ACGCTGACGCTCGTCTACTT & GAAAGTGCCAAAGCTGGATT \\
\hline & Inner set & TGGTTAGTGCCCTGTGCAGC & CAGCCAGCAGGTGGATACGA \\
\hline \multirow[t]{2}{*}{ hAQP12 } & Outer set & САACCCCACTGTGTCCCTGC & CCAGCCAGTACACCTGCACG \\
\hline & Inner set & CAGTGACCTGCACCTGCTGC & GACCAACAGAGCCACAGCGG \\
\hline \multirow[t]{2}{*}{$\mathrm{mAQP3}$} & Outer set & TGATGAATCGTTGTGGGGAG & CAGGAGTGGGGAGACAATGG \\
\hline & Inner set & TGGCTTCCTCACCATCAACT & GGCCCAGATTGCATCGTAGT \\
\hline \multirow[t]{2}{*}{$\mathrm{mAQP7}$} & Outer set & TGTTTGGCCTTGGTTCCGTG & CCACCAGTTGTTTCCGGCTC \\
\hline & Inner set & CAGCTATCTCGGTGTCAACTT & GAAGATAGGTGGCAAAAATGT \\
\hline $\mathrm{mAQP3}$ & For qPCR & AACCCTGCCCGTGACTTTGGA & CGAAGACACCAGCGATGGAACC \\
\hline $\mathrm{mAQP7}$ & For qPCR & CCTTGGTTCCGTGGCTCACAT & CAGAGATGCCGCCTGCTACAT \\
\hline hAQP3 & For $\mathrm{qPCR}$ & ATCAACCTGGCCTTTGGCTT & TATTCCAGCACCCAAGAAGGCTT \\
\hline mGAPDH & For qPCR & CCCCAGCAAGGACACTGAGCAAGAG & GCCCCTCCTGTTATTATGGGGGTC \\
\hline hGAPDH & For $\mathrm{qPCR}$ & CAGGGCTGCTTTTAACTCTGG & TGGGTGGAATCATATTGGAACA \\
\hline
\end{tabular}

Table 1. Primers for Nested-PCR and qPCR (SYBR Green)

reverse transcription, using RT reagent Kit (TAKARA, Dalian, China). Nested PCR was used to increase the specificity of DNA amplification. Two sets of primers were used in two successive reactions. The first PCR was performed in $25 \mu \mathrm{l}$ reactions consisting of $2 \mu \mathrm{l}$ embryo cDNA, $12.5 \mu \mathrm{l}$ premix Taq (TAKARA), $9.5 \mu \mathrm{l}$ double-distilled water $\left(\mathrm{ddH}_{2} \mathrm{O}\right), 0.5 \mu \mathrm{l}$ sense primer and $0.5 \mu \mathrm{l}$ antisense primer. Reaction conditions were $95^{\circ} \mathrm{C}$ for $5 \mathrm{~min}$ followed by 30 cycles of amplification step $\left(95^{\circ} \mathrm{C}\right.$ for $30 \mathrm{~s}, 60^{\circ} \mathrm{C}$ for $30 \mathrm{~s}, 72^{\circ} \mathrm{C}$ for $\left.1 \mathrm{~min}\right)$. For the second PCR, $0.2 \mu \mathrm{l}$ of the first PCR production were amplified and the reaction profile was: $95^{\circ} \mathrm{C}$ for 30 $\mathrm{s}, 60^{\circ} \mathrm{C}$ for $30 \mathrm{~s}, 72^{\circ} \mathrm{C}$ for $30 \mathrm{~s}$, and for 40 cycles. For positive control samples, total RNA was extracted with Tri-zol regent (TAKARA) according to the manufacturer's instructions. PCR was conducted in $25 \mu$ reactions consisting of $2 \mu \mathrm{l} \mathrm{cDNA}, 12.5 \mu \mathrm{l}$ premix Taq (TAKARA), $9.5 \mu \mathrm{lddH}_{2} \mathrm{O}, 0.5 \mu \mathrm{l}$ sense primer and $0.5 \mu \mathrm{l}$ antisense primer in the following profile: $95^{\circ} \mathrm{C}$ for $30 \mathrm{~s}, 60^{\circ} \mathrm{C}$ for $30 \mathrm{~s}, 72^{\circ} \mathrm{C}$ for $30 \mathrm{~s}$, and for 35 cycles. Human positive control cDNAs were from Human MTC ${ }^{\mathrm{TM}}$ Panel I and Human MTC ${ }^{\mathrm{TM}}$ Panel II (Clontech, Palo Alto, CA, USA). Negative controls were set up using a complete PCR mix without cDNA. The primers are listed in Table 1. The PCR products were analyzed by $1.5 \%$ agarose gel electrophoresis and stained with ethidium bromide. RT-PCR was repeated at least three times.

qPCR was carried out with SYBR-Green premix Ex Taq (TAKARA) in an Applied Biosystems 7500 Fast (ABI, Carlsbad, CA, USA), using GAPDH as internal controls. The primers are listed in Table 1. qPCR was performed in a $20 \mu \mathrm{l}$ reaction system containing $10 \mu \mathrm{l}$ SYBR premix Ex Taq, $0.4 \mu \mathrm{l}$ sense and $0.4 \mu \mathrm{l}$ antisense primers, $0.4 \mu \mathrm{l}$ Dye II, $6.8 \mu \mathrm{l} \mathrm{ddH_{2 }} \mathrm{O}, 2.0 \mu \mathrm{l} \mathrm{cDNA}$. The thermal cycling conditions were: $95^{\circ} \mathrm{C}$ for $10 \mathrm{~s}, 95^{\circ} \mathrm{C}$ for $5 \mathrm{~s}, 60^{\circ} \mathrm{C}$ for $34 \mathrm{~s}$, and for 40 cycles. Data were analyzed by the comparative threshold cycle (CT) method [15]. 


\section{Cellular Physiology and Biochemistry}

Cell Physiol Biochem 2013;31:649-658

DOI: $10.1159 / 000350084$

Published oniıne: IVIay 06, 2013

(C) 2013 S. Karger AG, Basel

www.karger.com/cpb

Xiong/Tan/Xiong et al.: Aquaporins and Pre-implanted Embryonic Development

\section{Immunofluorescence analysis}

The embryos were washed three times with $1 \times$ phosphate-buffered saline (PBS) and fixed at room temperature for 20 min in PBS containing 4\% paraformaldehyde. The embryos were blocked in $1 \times$ PBS containing 5\% BSA and 1\% saponin (Sigma-Aldrich) for $30 \mathrm{~min}$, followed by incubation with rabbit polyclonal anti-AQP7 antibody or goat polyclonal anti-AQP3 antibody (Santa Cruz Biotechnology, Santa Cruz, CA, USA) at a 1:200 dilution at $4{ }^{\circ} \mathrm{C}$ overnight. After rinsing three times, the embryos were incubated with Alexa Fluor 488 goat anti-rabbit IgG or rabbit anti-goat IgG or Alexa Fluor 594 rabbit anti-goat IgG (Invitrogen, Carlsbad, CA) at 1:400 dilution for $30 \mathrm{~min}$. The nuclear status of embryos was evaluated by staining with $1 \mathrm{mg} / \mathrm{ml}$ of 4',6-diamidino-2-phenylinedole (Sigma-Aldrich) for $10 \mathrm{~min}$. For negative controls, we omitted primary antibodies. Finally, fluorescent images were analyzed by Zeiss LSM 510Meta laser scanning confocal microscope (Carl Zeiss Inc., Thornwood, NY, USA).

\section{Microinjection of siRNA in mouse two-cell embryos}

Mouse 2-cell embryos, which showed normal appearance, were collected and stored in HEPESbuffered HTF containing 10\% serum substitute supplement ( $\mathrm{pH} 7.4$ ), covered with mineral oil (SigmaAldrich) at $37^{\circ} \mathrm{C}$. Scrambled RNA (4390843, Ambion, Carlsbad, CA, USA), mouse AQP3 siRNA (S62527, sense: 5'- GGA UUG UUU UUG GGC UGU ATT-3'; antisense: 5'-UAC AGC CCA AAA CAA UCC CA-3', Ambion) or mouse $A Q P 7$ siRNA (S62538, sense: 5'-GCA GCU ACC ACC UAC UUA ATT-3'; antisense: 5'-UUA AGU AGG UGG UAG CUG CAG-3', Ambion) was dissolved in RNase-free water. An embryo in HEPES-buffered HTF was held with a holding pipette connected to a micromanipulator on an inverted microscope, and injected with $10 \mathrm{pl}$ scrambled RNA solution (1 pg/pl, as control), or $A Q P 3$ siRNA solution (1 pg/pl) or AQP7 siRNA solution (1 $\mathrm{pg} / \mathrm{pl}$ ) with an injection needle connected to another micromanipulator. Non-injected embryos were used as another control (untreated). The injected embryos with normal shape just after injection were cultured in $\mathrm{HTF}$ containing $10 \%$ serum substitute supplement under $5 \% \mathrm{CO}_{2}$ at $37^{\circ} \mathrm{C}$. Images of every group of embryos were recorded with a video recorder (OLYMPUS, Tokyo, Japan) at $0 \mathrm{~h}, 24 \mathrm{~h}$ and $48 \mathrm{~h}$, respectively. Twenty-four hours after injection, the mRNA expression levels of $A Q P 3$ and $A Q P 7$ were evaluated using qPCR, respectively. Twenty-four and forty-eight hours after injection, protein expression of AQP3 and AQP7 was examined using immunofluorescent staining, respectively.

\section{JAr cell and Ishikawa cell culture and siRNA interference}

Human choriocarcinoma (JAr) cells (American Type Culture Collection, HTB 144) and Ishikawa cell line (Shanghai Institutes for Biological Science) were used to make an in vitro attachment model and to mimic embryo implantation. Both two cell lines were grown in RPMI 1640 supplemented with $10 \%$ fetal calf serum at $37^{\circ} \mathrm{C}$ in humidified air containing $5 \% \mathrm{CO}_{2}$. Human AQP3 ( $h A Q P 3$ ) small interfering RNA (siRNA) duplexes (S1522, sense: 5'-GGA UCA AGC UGC CAU CUA TT-3'; antisense: 5'-UAG AUG GGC AGC UUG AUC CAG-3', Ambion) were used for RNA interference experiments. Scrambled RNA (4390843, Ambion) was used as a control oligonucleotide. JAr cells were plated in $10 \mathrm{~cm}$ plates or six-well plates at $40 \%$ confluence for transfection. The cells were transfected with $5 \mathrm{nM}$ siRNA with lipofectamine 2000, according to the manufacturer's guidelines (Invitrogen). The expression levels of AQP3 mRNA were examined with qPCR 24 $\mathrm{h}$ after transfection.

\section{Attachment assay of JAr spheroids to Ishikawa cells}

Multicellular spheroids of JAr cells were used as an in vitro attachment model, and applied to endometrial cell (Ishikawa cell) monolayers as described before [15]. Briefly, JAr cells at 40\% confluence in $10 \mathrm{~cm}$ plate were transfected with $h A Q P 3$ siRNA or scrambled RNA for $24 \mathrm{~h}$ and then made into JAr spheroids according to a standard procedure [16]. Ishikawa cells were plated in 12-well plates to achieve a confluent monolayer. The spheroids were transferred onto the surface of a confluent monolayer of Ishikawa cells. The cultures were maintained in the culture medium (RPMI 1640 medium $+10 \%$ FBS) for $1 \mathrm{~h}$. The 12-well plates were centrifuged with the cell-spheroid surface facing down at $10 \mathrm{~g}$ for $10 \mathrm{~min}$. The attached spheroids were counted under a light microscope, and, the percentage of the total number of spheroids used, was calculated.

\section{Statistical analysis}

Data were analyzed using the Statistical Package for Social Sciences (SPSS 17.0 for Windows). In all histograms, error bars represent the standard error of the mean (SEM). Statistical comparisons were made 


\section{Cellular Physiology and Biochemistry}

Cell Physiol Biochem 2013;31:649-658

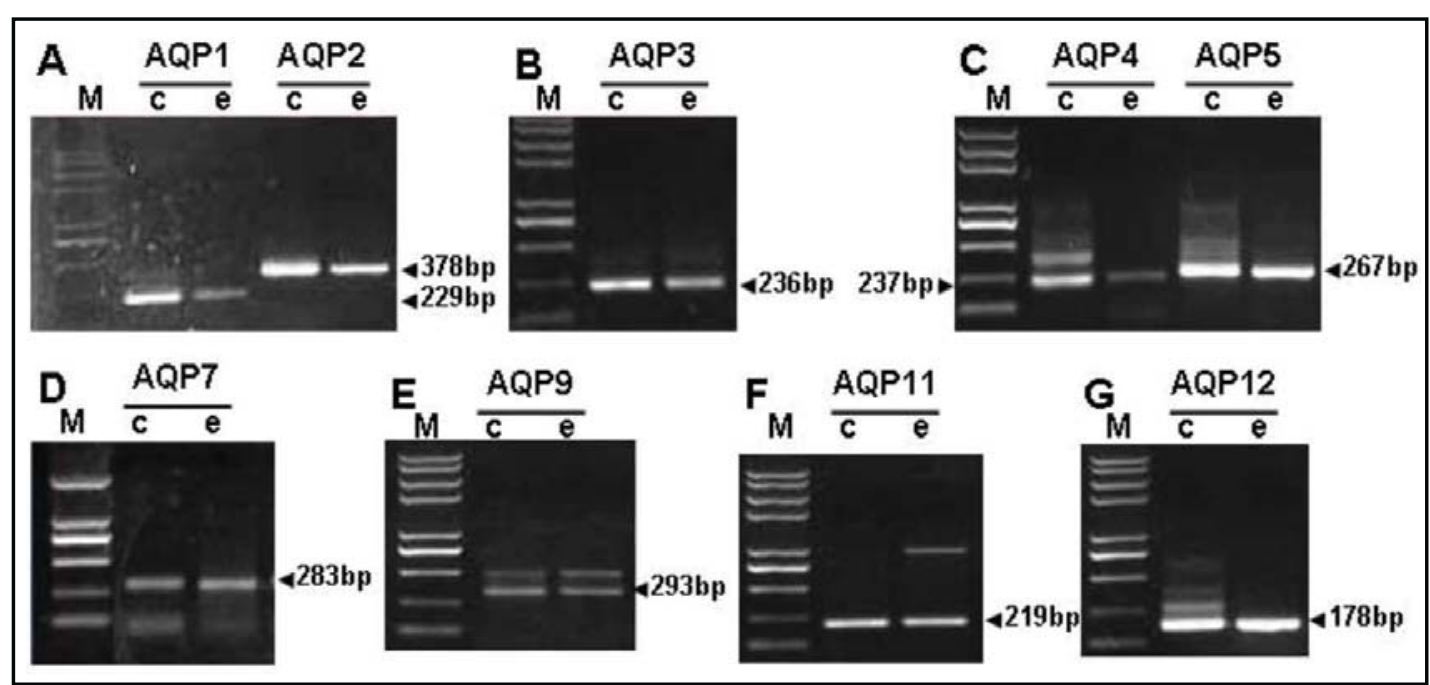

Fig. 1. Detection of AQP mRNAs in human embryos from the 2-cell to 8-cell stages. Figures A-G show that the mRNAs of AQPs 1, 2, 3, 4, 5, 7, 9, 11 and 12 were detected in human embryos. M, marker; c, positive control; e, embryo.

Fig. 2. Detection of AQP3 and AQP7 mRNAs in human and mouse oocytes and embryos from zygote to bastocyst stage. The mRNAs of AQP3 and AQP7 were expressed in human (A) and mouse (B) oocytes and embryos from zygote to bastocyst stage. $M$, marker; oo, oocyte: z, zygote; 2-c, 2-cell embryo; 4-c, 4-cell embryo; 8-c, 8-cell embryo; m, morula; b, blastocyst; nc, negative control.

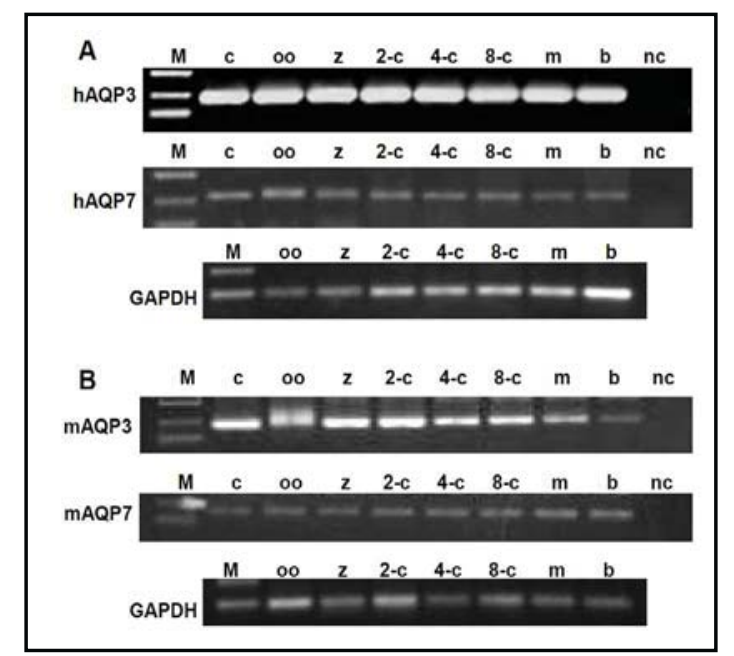

by Student's t-tests between two groups. One-way analysis of variance (ANOVA) and Turkey's post hoc tests were used to evaluate statistical significance of the difference between more than two groups. Statistical significance was set as $\mathrm{P}<0.05$.

\section{Results}

Expression of AQPs in human preimplantation embryos

To identify the expression profile of AQPs in human preimplantation embryos, we collected 30 human embryos at 2-cell to 8-cell stage to examine the mRNA expression of all 13 subtypes of AQPs with RT-PCR. We found that mRNAs of AQPs 1-5, 7, 9, 11-12 were expressed in human preimplantation embryos (Fig. 1). However, AQPs 0, 6, 8 and 10 mRNAs were not detected in human preimplantation embryos (data not shown).

Expression of AQP3 and AQP7 in human and mouse oocytes and preimplantation embryos from zygote to blastocyst stage

We examined the expression of AQP3 and AQP7, which are subtypes of aquaglyceroporin family, in human and mouse oocytes, and preimplantation embryos at different stages. We 


\section{Cellular Physiology and Biochemistry}

Cell Physiol Biochem 2013;31:649-658

\begin{tabular}{l|l}
\hline DOI: $10.1159 / 000350084$ & (C) 2013 S. Karger AG, Basel
\end{tabular}

www.karger.com/cpb

654

Fig. 3. Detection of $A Q P 3$ and AQP7 proteins in human and mouse oocytes and embryos from zygote to bastocyst stage. The proteins of AQP3 and AQP7 were expressed in human $(A)$ and mouse (B) oocytes and embryos from zygote to bastocyst stage. M, marker; oo, oocyte: $z$, zygote; 2 -c, 2-cell embryo; 4-c, 4-cell embryo; 8-c, 8-cell embryo; m, morula; b, blastocyst; NC, negative control.

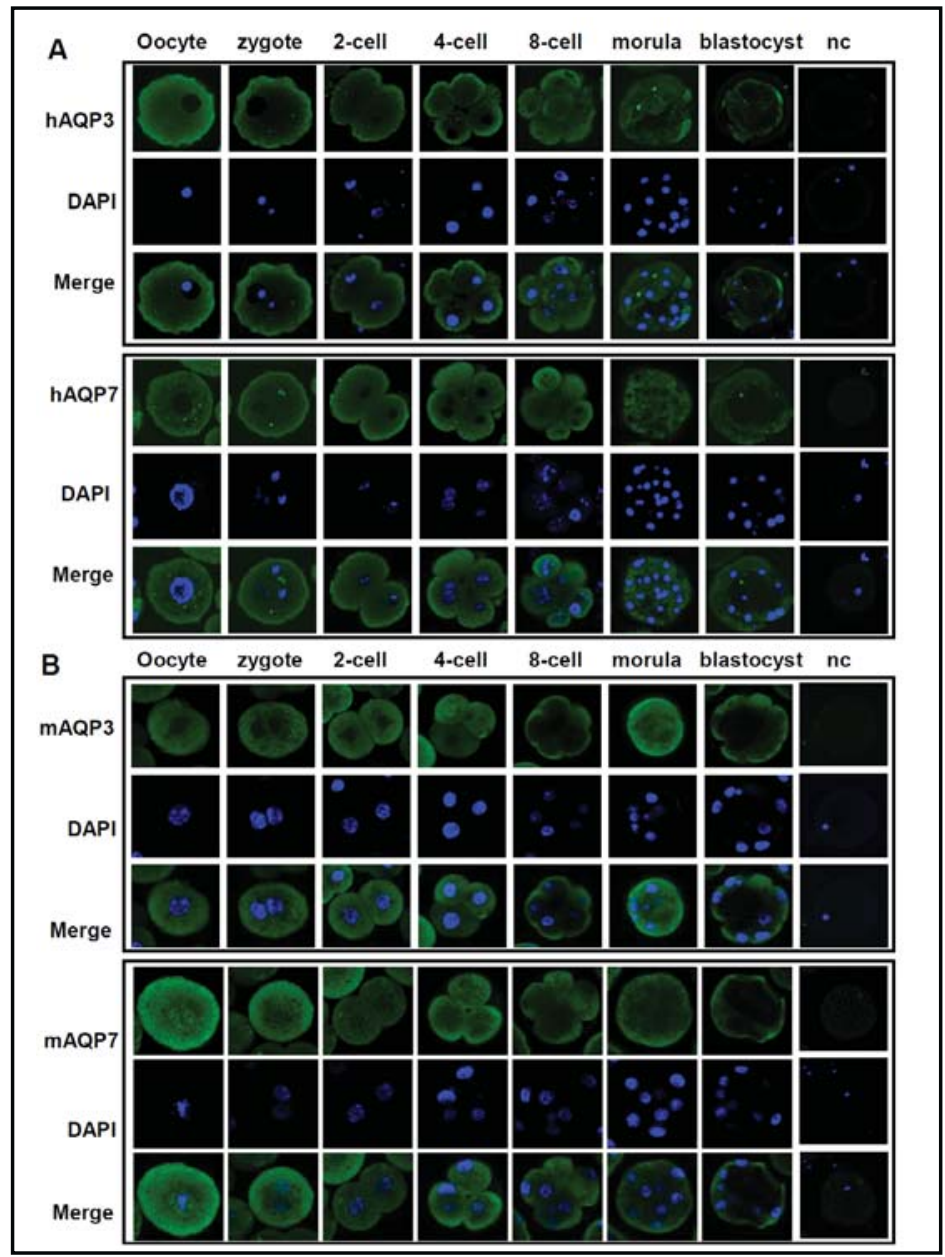

found that the mRNAs of AQP3 and AQP7 were expressed in human and mouse oocytes, and, preimplantation embryos from zygote to blastocyst stage (Fig. 2). Immunofluorescence analysis confirmed expression of AQP3 and AQP7 in human and mouse oocytes, and, preimplantation embryos from zygote to blastocyst stages (Fig. 3). The confocal images with immunocytochemical staining show that AQP3 and AQP7 (green) are mainly localized at the plasma membrane of human oocyte, and , preimplantation embryos from zygote to blastocyst stages (Fig. 3). However, AQP3 and AQP7 are mainly localized in the cytoplasm of mouse oocyte, and, preimplantation embryos from zygote to 2-cell stages, and at the plasma membrane of preimplantation embryos from 8-cell to blastocyst stages (Fig. 3).

\section{Inhibition of development of mouse preimplantation embryos pretreated with specific} siRNA targeting AQP3 and AQP7

We injected specific siRNA targeting $A Q P 3$ or $A Q P 7$ into each cell of mouse embryos at 2-cell stage, and, analyzed AQP3 and AQP7 expression using qPCR or immunofluorescence assay. Compared to embryos injected with scrambled siRNA, treatment of mouse preimplantation embryos with $A Q P 3$ siRNA or $A Q P 7$ siRNA for $24 \mathrm{~h}$ significantly reduced mRNA expression level of AQP3 by 86\% (Fig. 4A), and reduced mRNA expression level of AQP7 by 65\% (Fig. 4B), respectively. The immunofluorescence intensities of AQP3 (red) and AQP7 (green) were also lower in mouse preimplantation embryos $24 \mathrm{~h}$ or $48 \mathrm{~h}$ after injection with $A Q P 3$ siRNA and $A Q P 7$ siRNA, respectively, compared to the embryos injected with scrambled siRNA, (Fig. 4C).

We found that, after being cultured for $24 \mathrm{~h}$, the percentages of 2-cell embryo reaching to 4 -cell and 8 -cell stages were $33.6 \%$ and $47.5 \%$, respectively, in untreated group (Fig. 4D 
Fig. 4. The embryonic development of embryos injected with specific siRNA targeting AQP3 and AQP7, respectively. qPCR and immunofluorescence staining analyses show low expression of AQP3 and AQP7 in mouse embryos injected with $A Q P 3$ siRNA and $A Q P 7$ siRNA, respectively (A-C). The embryonic development was significantly inhibited in the embryos injected with $A Q P 3$ siRNA or $A Q P 7$ siRNA at the 2-cell stage $24 \mathrm{~h}$ or $48 \mathrm{~h}$ after injection (D). Summary data are shown in E (24 h) and F (48 h). Data are present as mean \pm SE. * and **, $P<0.05$ and $P<0.01$ compared to the untreated controls, respectively. \# and \#\#, $P<0.05$ and $P<0.01$ compared to the scrambled RNA group, respectively. $n$, number of repeated experiments.

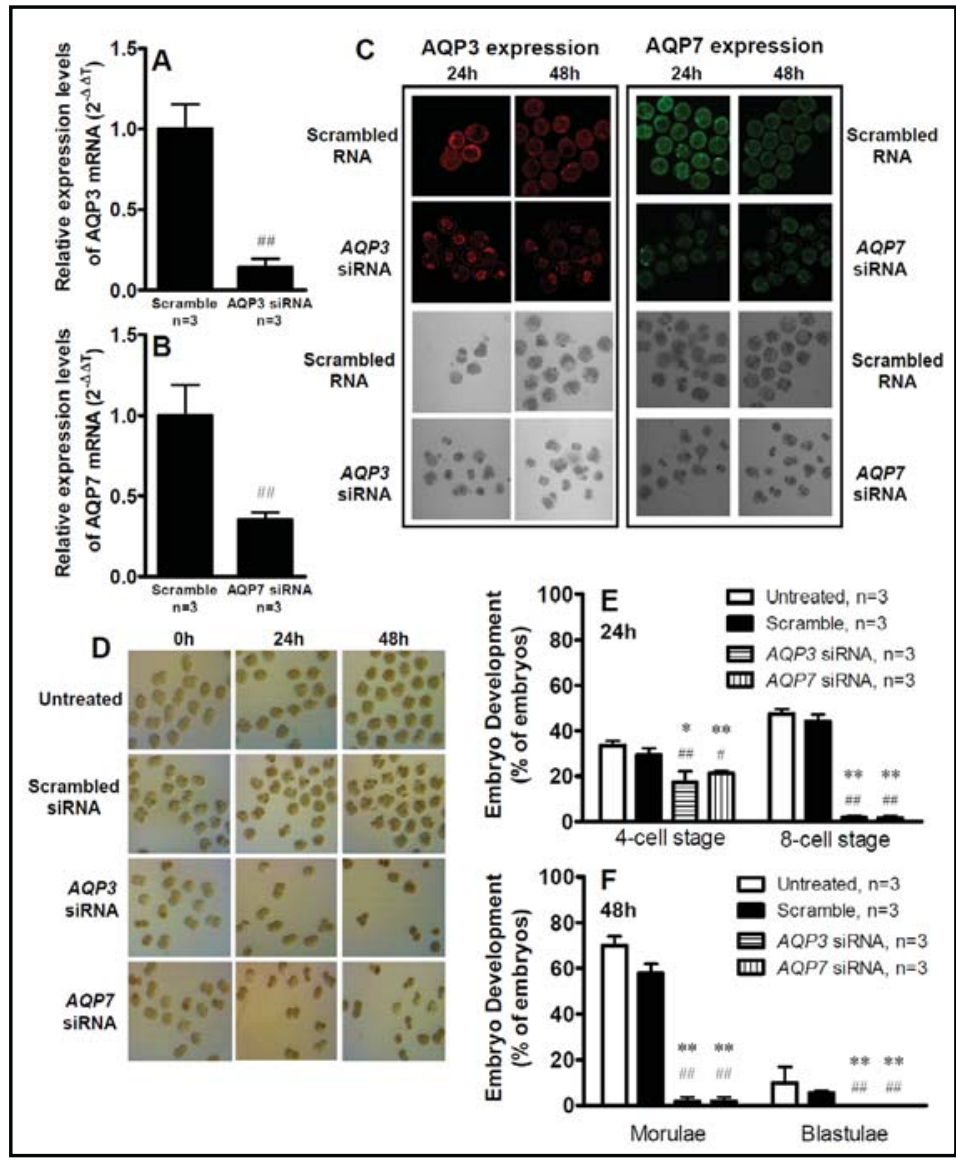

and E). After being cultured for $48 \mathrm{~h}$, the percentages of 2-cell embryo reaching to morula and blastula stages were $70.0 \%$ and $9.9 \%$, respectively, in untreated group (Fig. 4D and F). There was no significant difference in the percentages of 2-cell embryos reaching to 4-cell, 8-cell, morula or blastula stage between untreated and scrambled groups (Fig. 4D, $\mathrm{E}$ and F). However, the percentages of 2-cell embryos reaching to 4-cell, 8-cell, morula and blastula stage in $A Q P 3$ siRNA-treated group were $17.2 \%, 1.75 \%, 1.75 \%$ and $0 \%$, respectively, significantly lower than those in untreated and scrambled groups (Fig. 4D, E and F). The percentage of 2-cell embryos reaching to 4-cell, 8-cell, morula and blastula stage in $A Q P 7$ siRNA-treated group were $21.3 \%, 1.5 \%, 1.75 \%$ and $0 \%$, respectively, also significantly lower than those in untreated and scrambled groups (Fig. 4D, E and F).

\section{Attachment rate of JAr spheroid pretreated with specific siRNA targeting AQP3}

To determine the roles of embryonic AQP3 and/or AQP7 in embryo implantation, we employed a JAr spheroid attachment assay. JAr spheroids represent an appropriate embryolike model for studies mimicking implantation [17]. We found that JAr cells expressed AQP3 (Fig. 5A), but not AQP7 (Fig. 5B). Although treatment of JAr cells with AQP3 siRNA significantly reduced AQP3 mRNA levels in JAr cells (Fig. 5C), knockdown of AQP3 in JAr cells did not alter the attachment rate of JAr spheroids to endometrial cells (Ishikawa cells), compared to JAr spheroids transfected with scrambled siRNA (Fig. 5D and E).

\section{Discussion}

In the present study, we have demonstrated that the mRNAs of AQPs 1-5, 7, 9, 11-12 were expressed in human preimplantation embryos. We also found that AQP3 and AQP7 were expressed in human oocytes and preimplantation embryos from zygote to blastocyst 
Fig. 5. Effects of $A Q P 3$ knockdown in JAr cells on JAr spheroid attachment. RT-PCR analysis shows that JAr cells expressed AQP3 mRNA (A), not AQP7 mRNA (B). AQP3 mRNA level in JAr cells transfected with specific siRNA targeted $A Q P 3$ was significantly lower than scrambled siRNA treatment (C). Arrow in D indicates the JAr spheroid attached to human endometrial cells. E: The JAr spheroid attachment rates in scrambled group and $A Q P 3$ siRNA group. Data are presented as mean $\pm \mathrm{SE}$. **, $\mathrm{P}<0.05$ or $\mathrm{P}<0.01$, compared with the scrambled RNA group. $n$, number of repeated experiments.
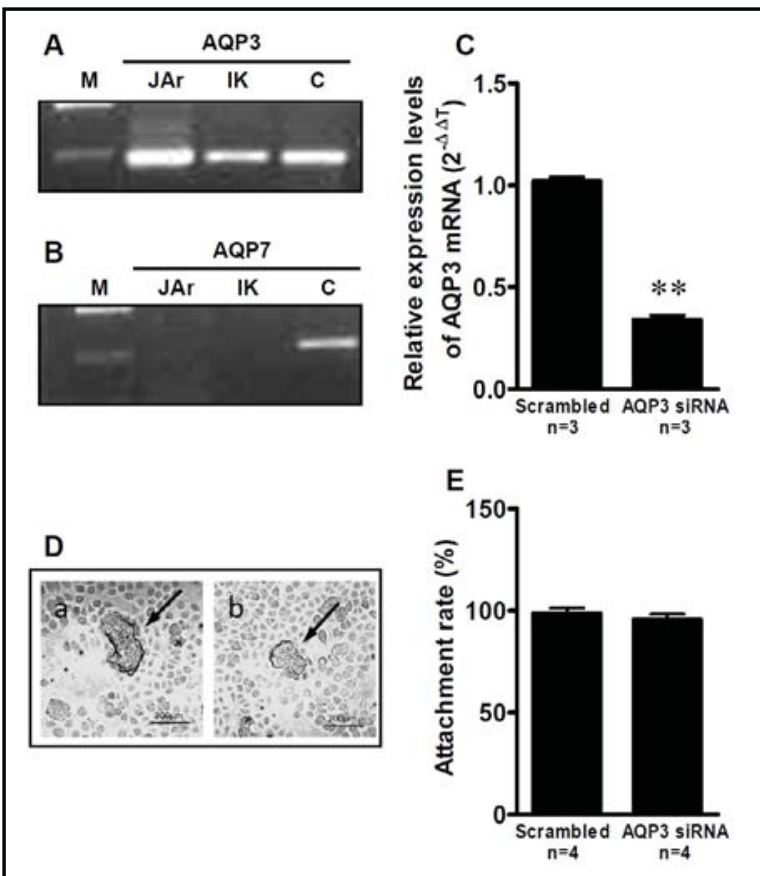

stages. In mice, knockdown of embryonic AQP3 and AQP7 significantly inhibited mouse preimplantation embryonic development though, knockdown of AQP3 in JAr cells did not affect the JAr spheroid, an appropriate embryo-like model, attachment rate.

Up to date, no other published studies have investigated the expression profile of AQPs in human embryos. A previous study showed that AQPs 1, 3 and 5-9 were expressed in mouse embryos [7], and another study did not detect AQPs 1, 2, 4, 5 and 6 in mouse oocytes and embryos at any stage [8]. Our results showed that human preimplantation embryos expressed mRNAs encoding AQPs 1-5, 7, 9 and 11-12. This result suggests that the expression profile of human AQPs may be different from the finding in mouse embryos. We believe that our data will help us to understand the roles of AQPs in the regulation of transport of water and other small molecules in embryos of human and other mammals.

The roles of AQP3 in oocyte and embryo cryopreservation have been investigated because AQP3 is an aquaglyceroporin which might facilitate cryoprotectants such as ethylene glycol and dimethyl sulfoxide. AQP3 was demonstrated to mediate water and cryoprotectant movement and regulated embryo apoptosis during embryo cryopreservation [18-21]. In the present study, we demonstrated expression of AQP3 and AQP7 mRNAs and proteins in mouse oocytes and preimplantation embryos at all stages. Knockdown of AQP3 or AQP7 expression in mouse embryos at 2-cell stage significantly inhibited embryonic development, suggesting an important role of AQP3 or AQP7 in embryonic development. Down-regulation of AQP3 has been shown to be involved in impaired function of several cell types including oocytes, embryos and somatic cells. Knockout of AQP3 in mouse zygotes significantly decreased water and cryoprotectant permeability [21]. Impaired proliferation of epidermal cells was observed in AQP3 null mice [22]. Reduced AQP3 also inhibited the growth of human esophageal and oral squamous cell carcinoma [23]. On the other hand, an association of increased AQP3 expression with oocyte maturation was demonstrated [24]. Overexpression of AQP3 in mouse oocytes improved the oocyte survival during cryopreservation [11].

The mechanisms underlying the inhibition of embryonic development by knockdown of AQP3 or AQP7 may involve both the altered water- and glycerol-transporting functions of AQP3 and AQP7. Glycerol acts as energy substrate for glycolysis, and its metabolism is catalyzed by the glycerol kinase that phosphorylates glycerol to glycerol-3-phosphate, which is one of the key metabolic intermediates for ATP production [25]. It has been shown that glycerol kinase was expressed in bovine embryos at all the stages, and its expression 


\section{Cellular Physiology and Biochemistry}

Cell Physiol Biochem 2013;31:649-658

\begin{tabular}{l|l}
\hline DOI: $10.1159 / 000350084$ & (c) 2013 S. Karger AG, Basel
\end{tabular}

Publisned onine: IVlay 06, 2013

www.karger.com/cpb

Xiong/Tan/Xiong et al.: Aquaporins and Pre-implanted Embryonic Development

significantly increased at the morula stage [26]. Down-regulation of AQP3 expression in keratinocytes reduced glycerol transport across membrane and cell proliferation, and glycerol supplementation could correct the reduced cell proliferation [22]. Further experiments are needed to investigate the link between AQP3 or AQP7 and preimplantation embryonic development.

It has been demonstrated that $A Q P 3$ knockout mice could develop to term [22, 27]. In the present study, we injected specific siRNA targeting $A Q P 3$ or $A Q P 7$ into embryos at 2-cell stage. The knockdown of AQP3 or AQP7 expression occurred in preimplantation embryonic cells from the 2-cell stage embryo onward. The down-regulation of AQP3 and AQP7 might be not completely compensated by other water channels or other membrane proteins from the 2-cell stage. On the other hand, the methods used to knock down AQP3 in present study and knock out $A Q P 3$ in other studies were different, which may be another reason for the different results between our and other studies. These possibilities are needed to be clarified in our further studies. However, we found that knockdown of AQP3 expression in JAr cells did not affect the attachment rate of JAr spheroid to endometrial cells, suggesting that downregulation of AQP3 in embryos might not affect embryo implantation.

In conclusion, our data provide, to our knowledge for the first time, the expression profile of AQPs in human preimplantation embryos. AQP3 or AQP7 deficiency in the embryos from the 2-cell stage onward may inhibit embryonic development. Our findings suggest that the maintenance of normal AQP3 and AQP7 levels in preimplantation embryos may be essential for early embryonic development.

\section{Disclosure Statement}

The authors have no conflict of interest to disclose.

\section{Acknowledgements}

Authors thank Dr. Martin Quinn from London, UK for his reading and editing this manuscript. Authors also thank Dr. Yu-Li Qian in the IVF laboratory, Women's Hospital, Zhejiang University, for his help in collection of human oocytes and embryos.

Human embryo expresses aquaporins (AQPs) 1-5, 7, 9, 11-12, and AQP3 and AQP7 are essential for early embryonic development.

This work was supported by National Basic Foundation of China (NO. 2011CB944502 and NO. 2012CB944903), National Key Technology R\&D Program (NO. 2012BAI32B00), and the Public Welfare Technology Application Research Project of Zhejiang Province (2010C33167, 2010C13028).

\section{References}

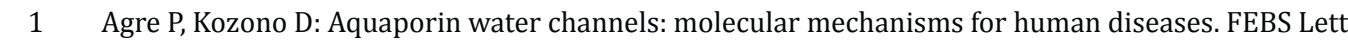
2003;555:72-78.

$\longrightarrow 2$ Benga G: The first discovered water channel protein, later called aquaporin 1: Molecular characteristics, functions and medical implications. Mol Aspects Med 2012;33:518-534.

- Zardoya R: Phylogeny and evolution of the major intrinsic protein family. Biol Cell 2005;97:397-414.

-4 Nozaki K, Ishii D, Ishibashi K: Intracellular aquaporins: clues for intracellular water transport? Pflugers Arch 2008;456:701-707.

5 Huang HF, He RH, Sun CC, Zhang Y, Meng QX, Ma YY: Function of aquaporins in female and male reproductive systems. Hum Reprod Update 2006;12:785-795.

-6 Zhang D, Tan YJ, Qu F, Sheng JZ, Huang HF: Functions of water channels in male and female reproductive systems. Mol Aspects Med 2012;33:676-690. 


\section{Cellular Physiology and Biochemistry}

Cell Physiol Biochem 2013;31:649-658

\begin{tabular}{l|l}
\hline DOI: $10.1159 / 000350084$ & (C) 2013 S. Karger AG, Basel
\end{tabular}

Published onlıne: IVay U6, $2013 \quad$ www.karger.com/cpb

Xiong/Tan/Xiong et al.: Aquaporins and Pre-implanted Embryonic Development

7 Offenberg H, Barcroft LC, Caveney A, Viuff D, Thomsen PD, Watson AJ: mRNAs encoding aquaporins are present during murine preimplantation development. Mol Reprod Dev 2000;57:323-330.

-8 Edashige K, Sakamoto M, Kasai M: Expression of mRNAs of the aquaporin family in mouse oocytes and embryos. Cryobiology 2000;40:171-175.

9 Seki S, Edashige K, Wada S, Mazur P: Effect of the expression of aquaporins 1 and 3 in mouse oocytes and compacted eight-cell embryos on the nucleation temperature for intracellular ice formation. Reproduction 2011;142:505-515.

10 Edashige K, Tanaka M, Ichimaru N, Ota S, Yazawa K, Higashino Y, Sakamoto M, Yamaji Y, Kuwano T, Valdez DM Jr, Kleinhans FW, Kasai M: Channel-dependent permeation of water and glycerol in mouse morulae. Biol Reprod 2006;74:625-632.

11 Edashige K, Yamaji Y, Kleinhans FW, Kasai M: Artificial expression of aquaporin-3 improves the survival of mouse oocytes after cryopreservation. Biol Reprod 2003;68:87-94.

$\checkmark 12$ Cho YS, Svelto M, Calamita G: Possible functional implications of aquaporin water channels in reproductive physiology and medically assisted procreation. Cell Mol Biol (Noisy-le-grand) 2003;49:515-519.

13 Meng QX, Gao HJ, Xu CM, Dong MY, Sheng X, Sheng JZ, Huang HF: Reduced expression and function of aquaporin-3 in mouse metaphase-II oocytes induced by controlled ovarian hyperstimulation were associated with subsequent low fertilization rate. Cell Physiol Biochem 2008;21:123-128.

14 Tan YJ, Xiong Y, Ding GL, Zhang D, Meng Y, Huang HF, Sheng JZ: Cryoprotectants up-regulate expression of mouse oocyte AQP7, which facilitates water diffusion during cryopreservation. Fertil Steril 2013;99:14281435.

-15 Liu XM, Ding GL, Jiang Y, Pan HJ, Zhang D, Wang TT, Zhang RJ, Shu J, Sheng JZ, Huang HF: Down-regulation of S100A11, a calcium-binding protein, in human endometrium may cause reproductive failure. J Clin Endocrinol Metab 2012;97:3672-3683.

-16 Grümmer R, Hohn HP, Mareel MM, Denker HW: Adhesion and invasion of three human choriocarcinoma cell lines into human endometrium in a three-dimensional organ culture system. Placenta 1994;15:411429.

17 Wang H, Bocca S, Anderson S, Yu L, Rhavi BS, Horcajadas J, Oehninger S: Sex steroids regulate epithelialstromal cell cross-talk and trophoblast attachment-invasion in a three-dimensional human endometrial culture system. Tissue Eng Part C Methods 2013; doi:10.1089/ten.tec.2012.0616.

18 Yamaji Y, Seki S, Matsukawa K, Koshimoto C, Kasai M, Edashige K: Developmental ability of vitrified mouse oocytes expressing water channels. J Reprod Dev 2011;57:403-408.

19 Kuzmany A, Havlicek V, Wrenzycki C, Wilkening S, Brem G, Besenfelder U: Expression of mRNA, before and after freezing, in bovine blastocysts cultured under different conditions. Theriogenology 2011;75:482-494.

20 Bell CE, Larivière NM, Watson PH, Watson AJ: Mitogen-activated protein kinase (MAPK) pathways mediate embryonic responses to culture medium osmolarity by regulating Aquaporin 3 and 9 expression and localization, as well as embryonic apoptosis. Hum Reprod 2009;24:1373-1386.

-21 Edashige K, Ohta S, Tanaka M, Kuwano T, Valdez DM Jr, Hara T, Jin B, Takahashi S, Seki S, Koshimoto C, Kasai M: The role of aquaporin 3 in the movement of water and cryoprotectants in mouse morulae. Biol Reprod 2007;77:365-375.

-22 Hara-Chikuma M, Verkman AS: Aquaporin-3 facilitates epidermal cell migration and proliferation during wound healing. J Mol Med (Berl) 2008;86:221-231.

-23 Kusayama M, Wada K, Nagata M, Ishimoto S, Takahashi H, Yoneda M, Nakajima A, Okura M, Kogo M, Kamisaki Y: Critical role of aquaporin 3 on growth of human esophageal and oral squamous cell carcinoma. Cancer Sci 2011;102:1128-1136.

-24 Jo JW, Jee BC, Suh CS, Kim SH, Choi YM, Kim JG, Moon SY: Effect of maturation on the expression of aquaporin 3 in mouse oocyte. Zygote 2011;19:9-14.

25 Brisson D, Vohl MC, St-Pierre J, Hudson TJ, Gaudet D: Glycerol: a neglected variable in metabolic processes? Bioessays 2001;23:534-542.

26 Okawara S, Hamano S, Tetsuka M: Bovine oocytes and early embryos express mRNA encoding glycerol kinase but addition of glycerol to the culture media interferes with oocyte maturation. J Reprod Dev 2009;55:177-182.

27 Ma T, Song Y, Yang B, Gillespie A, Carlson EJ, Epstein CJ, Verkman AS: Nephrogenic diabetes insipidus in mice lacking aquaporin-3 water channels. Proc Natl Acad Sci USA 2000;97:4386-4391. 